\title{
THE ATMOSPHERIC COMPENSATION COMPONENT OF A LANDSAT LAND SURFACE TEMPERATURE (LST) PRODUCT: ASSESSMENT OF ERRORS EXPECTED FOR A NORTH AMERICAN TEST PRODUCT
}

\author{
M. J. Cook, J. R. Schott* \\ Rochester Institute of Technology, Chester F Carlson Center for Imaging Science, \\ 54 Lomb Memorial Drive, Rochester, New York 14623 - schott@ cis.rit.edu
}

KEY WORDS: Landsat, Surface Temperature, Atmospheric Compensation

\begin{abstract}
:
The Landsat archive of thermal data (Landsats 4, 5 and 7) has gone through a rigorous calibration assessment and update. However, in order to be useful to most users the calibrated sensor reaching radiance must be corrected to surface temperatures by first compensating for atmospheric effects and then emissivity variations. The USGS is exploring the possibility of producing a LST product through a joint program with RIT (the atmospheric compensation component) and JPL (the emissivity compensation component). This paper addresses the atmospheric compensation component for an initial North American pilot study. In particular, the results of a comparison of retrieved water surface temperature (where emissivity is well known) and truth temperatures for over 800 sites are presented. The errors are broken down by cloud conditions with extremely good results for cloud-free conditions (errors less than $1 \mathrm{~K}$ ). The results of the error assessment for North America by cloud class are presented along with a discussion of potential quality data for a LST product. An initial assessment of the LST errors observed for Landsat 8 bands 10 and 11 are also presented. The next steps on this effort include testing of a global atmospheric compensation approach and full integration of the atmospheric and emissivity compensation tools into an operational LST product.
\end{abstract}

\section{INTRODUCTION}

Landsat is the longest set of continuously acquired, moderate resolution satellite imagery. The fourth satellite in the family, Landsat 4, was the first to capture single thermal band imagery beginning in 1982, followed by Landsat 5 and Landsat 7. Landsat 8, launched in February 2013, is the first to capture multiple thermal bands. With these four instruments, there are currently more than four million Landsat thermal images in the archive and between 990 and 1090 scenes acquired each day. The entire archive (with the exception of Landsat 8 for which work is currently ongoing) has been radiometrically calibrated and characterized so that the sensor reaching radiance values are well known. However, radiance values are difficult to interpret, so this large archive of thermal data is under utilized.

For most users, thermal data is more useful as a temperature value. The temperature of the land surface, the first interface between the atmosphere and solid earth, is a useful metric across a large number of applications, including climatology, numerical weather prediction, and agriculture. LST is difficult to measure without altering the temperature of the surface and, for many investigations, these values are useful over large areas and long time scales, making LST products derived from satellite imagery an obvious answer. Most LST products are generated from multiple thermal bands using a split window approach, which utilizes the differential absorption between adjacent thermal bands (Wan and Dozier, 1996). Conversion from thermal radiance to land surface temperature with only a single thermal band requires complete characterization of the atmosphere and known surface emissivity.

Our colleagues at the Jet Propulsion Laboratory (JPL) are working to develop a high spatial resolution $(100 \mathrm{~m})$ surface emissivity product form the Advanced Spaceborne Thermal Emission and Reflection (ASTER) radiometer. Currently available is

${ }^{*}$ Corresponding author. the North American ASTER Land Surface Emissivity Database (NAALSED), but plans are underway to extend this to include a dataset with global coverage (Hulley and Hook, 2009). Work discussed in this paper assumes the availability of an emissivity product and focuses on the generation and error analysis of the atmospheric compensation component. Access to high quality reanalysis data makes the atmospheric characterization for a single band product feasible.

Generating radiative transfer parameters from atmospheric compensation is well understood, but the methodology presented here incorporates and interpolates the reanalysis data and the automates the process to generate unique values at every pixel in the scene. Previous studies indicate that the atmospheric compensation will be the largest source of error (i.e. larger error contributions than emissivity). We begin with an initial study and validation over North America, to be combined with NAALSED, and an analysis of the expected error and current suggestion for a confidence metric. This will later be extended to a global product.

\section{BACKGROUND}

The goal of this work is to generate, for every pixel in any Landsat scene in the archive, the radiative transfer parameters from the atmospheric compensation and the expected uncertainty. This section aims to summarize the necessary tools and datasets used in the methodology to determine the transmission, upwelled radiance, and downwelled radiance for each pixel in any scene.

\subsection{MODerate Resolution Atmospheric TRANsmission Ra- diative Transfer Code}

The MODerate resolution atmospheric TRANsmission (MODTRAN) radiative transfer code, developed from LOWTRAN, utilizes a propagation model that assumes the atmosphere is divided into a number of homogenous layers (Schott, 2007). Created by Spectral Sciences Inc. and the United States Airforce, 
MODTRAN solves the radiative transfer equation to characterize reflections, emissions, and transmissions, among other outputs (SSI, 2012). While the applications and uses of MODTRAN are far-reaching, for the purpose of this work, complete characterizations of the atmosphere (pressure, temperature, and relative humidity) are input into MODTRAN, and the spectral outputs (wavelength and radiance) are used to calculate the radiative transfer parameters.

\subsection{The North American Regional Reanalysis Dataset}

The atmospheric profiles input into MODTRAN are subset from the North American Regional Reanalysis Dataset. Reanalysis, or retrospective-analysis, is the process of using observing systems with numerical models to generate variables not easily observed or measured in a regular and consistent spatial and temporal set (Rienecker and Gass, 2013). The National Center for Environmental Prediction (NCEP) produces the NARR dataset using radiosondes, dropsondes, pibals, aircraft and surface data, and cloud drift winds among other inputs. This work utilizes profiles provided 8 times daily at 29 specific pressure levels, with approximately $0.3^{\circ}$ (approximately $32 \mathrm{~km}$ at the lowest latitude) spacing covering North America in a 349 by 277 array (Shafran, 2007). The geopotential height [gpm] and specific humidity $[\mathrm{kg} / \mathrm{kg}]$ profiles are converted to geometric height $[\mathrm{km}]$ and relative humidity $[\%]$ and input, along with the temperature profile $[\mathrm{K}]$, into MODTRAN.

\subsection{The Governing Equation}

A governing equation contains all of the components that are relevant to the sensor reaching radiance. Equation 1 shows the governing equation for a single Landsat thermal band, where $L_{\text {obs } \lambda \text { eff }}$ is the effective sensor reaching spectral radiance, $L_{T \lambda e f f}$ is the effective spectral radiance due to temperature, $\epsilon$ is the surface emissivity of the pixel of interest, $\tau$ is the transmission, $L_{u \lambda e f f}$ is the upwelled effective spectral radiance, and $L_{d \lambda e f f}$ is the downwelled effective spectral radiance.

$$
L_{o b s \lambda e f f}=\left(L_{T \lambda e f f} \epsilon+(1-\epsilon) L_{d \lambda e f f}\right) \tau+L_{u \lambda e f f}
$$

The radiance due to temperature is the term that we wish to isolate; this can be inverted to temperature through Plancks Equation, as shown in Equation 2. Because this equation is not directly invertible, we use a look up table. Given a radiance due to temperature, we invert to temperature with a two point linear interpolation using a look up table in $1 \mathrm{~K}$ increments.

$$
L_{T \lambda e f f}=\frac{M_{\lambda e f f}}{\pi}=\frac{\int\left[2 h c^{2} \lambda^{-5}\left(e^{\frac{h c}{\lambda k T}}-1\right)^{-1}\right] R(\lambda) d \lambda}{\int R(\lambda) d \lambda}
$$

Therefore, we need to solve for all other components of the governing equation in order to isolate and determine the radiance due to temperature. The observed radiance, $L_{o b s \lambda e f f}$, is generated from the digital number provided in the Landsat thermal band and the corresponding calibration coefficients in the scene metadata. For the case of this work, we assume that Landsats 4,5 , and 7 are characterized and calibrated and that this radiance value can be trusted without independent validation (Barsi et al., 2003) and (Padula et al., 2010). We also assume the incorporation of the ASTER derived emissivity, which leaves only the transmission, upwelled radiance, and downwelled radiance.
These effective spectral values are those that will be generated for every pixel in the Landsat scene through the methodology described in Section 3. We also believe that this atmospheric compensation process contributes to the limiting factor in uncertainty in the final LST product, so the confidence metric associated with the atmospheric compensation is discussed in Section 4.

Effective spectral radiance values indicate that the spectral response function of the sensor being used, which differs slightly for each Landsat instrument, has been accounted for over the spectral range of sensitivity. This is shown in Equation 3, where $L_{\lambda}$ could be any radiance value we consider, such as observed, upwelled, or downwelled. Effective spectral radiance values have units of $\mathrm{Wm}^{-2} \mathrm{sr}^{-1} \mu \mathrm{m}^{-1}$. All effective spectral radiance values are considered in this process and the explicit notation will be dropped from here forward.

$$
L=L_{\lambda e f f}=\frac{\int L_{\lambda} R(\lambda) d \lambda}{\int R(\lambda) d \lambda}
$$

\section{METHODOLOGY}

This methodology aims to process a single Landsat scene and generate the radiative transfer parameters, transmission, upwelled radiance, and downwelled radiance, for every pixel in the scene. This assumes the availability and incorporation of a digital elevation model providing the elevation of each pixel in the scene. For a particular Landsat scene, the acquisition time of the scene is identified, and the appropriate NARR variables (geopotential height, specific humidity, and temperature) at the time samples before and after this acquisition time are subset to include only locations pertinent to the current scene. This generally results in between 110 and 150 points for each scene. Each variable is temporally interpolated to the Landsat acquisition time using a simple two point linear interpolation. For example, NARR samples from $12 \mathrm{Z}$ and $15 \mathrm{Z}$ would be linearly interpolated to a Landsat acquisition time of $14.3 \mathrm{Z}$.

At each NARR point in the scene, the radiative transfer parameters are generated for nine separate ground altitudes using MODTRAN. For each MODTRAN run, we input a boundary temperature (with corresponding radiance due to temperature, $\mathrm{L}_{T}$ ) and albedo (with corresponding emissivty, $\epsilon$ ), and MODTRAN outputs an array of spectral observed radiance at the sensor for the atmosphere being characterized $\left(L_{o b s}\right)$. As shown in Equation 4 , when $\epsilon=1$, the governing equation reduces to a simple linear equation where the slope is equal to the transmission and the intercept is equal to the upwelled radiance. After two MODTRAN runs, two data points $\left(L_{T_{1}}, L_{o b s_{1}}\right)$ and $\left(L_{T_{2}}, L_{o b s_{2}}\right)$ can be used in a linear regression to generate transmission and upwelled radiance for the current atmosphere. With these values, a third MODTRAN run generates a final data point with some emissivity less than one, such that the governing equation can be solved for downwelled radiance as shown in Equation 5. The boundary temperature for this third MODTRAN run is equal to the air temperature of the lowest layer of the atmosphere, and the emissivity is set to 0.9 . These operations are performed at nine separate ground altitudes at every NARR point pertinent to the current scene.

$$
\begin{gathered}
L_{o b s}=L_{T} \tau+L_{u} \\
L_{d}=\frac{\frac{L_{o b s}-L_{u}}{\tau}-L_{T} \epsilon}{1-\epsilon}
\end{gathered}
$$


After radiative transfer parameters have been generated such that the data cube encompasses an entire Landsat scene, operations must be performed at every pixel in the scene in order to generate unique radiative transfer parameters for each pixel. For each pixel, the four most relevant NARR points (closest point in each direction) are selected and the radiative transfer parameters are piecewise linearly interpolated to the height of the current pixel at those four NARR points. This results in the radiative transfer parameters at the four closest NARR points associated with the appropriate elevation; the final step is to interpolate these values to the location of the current pixel. Shepards method was chosen as an inverse distance weighting function to interpolate the three radiative transfer parameters to the appropriate location for each pixel; for each of the four NARR points, a distance is calculated $\left(d_{i}\right)$ based on the NARR point location $\left(x_{i}, y_{i}\right)$ and the pixel location $(\mathrm{x}, \mathrm{y})$ [Equation 6] and weighting values $\left(w_{i}\right)$ are calculated based on these distances [Equation 7]. These weights are used to interpolate the original values at each location $\left(f_{i}\right)$ to the new interpolated value at the pixel location (F) [Equation 8] (Shepard, 1968).

$$
\begin{gathered}
d_{i}=\sqrt{\left(x-x_{i}\right)^{2}+\left(y-y_{1}\right)^{2}} \\
w_{i}=\frac{d_{i}^{-p}}{\sum_{j=1}^{n} d_{j}^{-p}} \\
F(x, y)=\sum_{i=1}^{n} w_{i} f_{i}
\end{gathered}
$$

This methodology results in the transmission, upwelled radiance, and downwelled radiance at every pixel in the Landsat scene.

\section{RESULTS}

\subsection{Ground Truth Data}

In order to validate our methodology, we compare the predicted temperatures from our process to ground truth water temperatures. We choose to validate against water temperatures because the emissivity of water is well known and because an instrument can be submerged and acclimated, so the temperature of water can be measured without the act of measuring altering the results. The measured bulk temperature still needs to be corrected to the skin or surface temperature of the water. Ground truth data for this study was provided for two sites by the Jet Propulsion Laboratory (Hook et al., 2007) and also derived from NOAA buoys from around the country through an accepted skin temperature correction method (Padula et al., 2010). Figure 1 shows the distribution of sites used throughout the United States; sites were chosen to capture a variety of elevation, climate, and atmosphere and images were chosen to capture a variety of season and atmospheric condition.

\subsection{Validation of Methodology}

In order to validate the methodology, initially only cloud free scenes were processed. A validation dataset of 259 cloud free Landsat 5 scenes, each containing one of the validation sites shown above, were processed and the predicted temperature at the site of the validation measurement was compared to the ground truth temperature provided by JPL or determined from the NOAA buoy measurements. Error was calculated using Equation 9; note that a

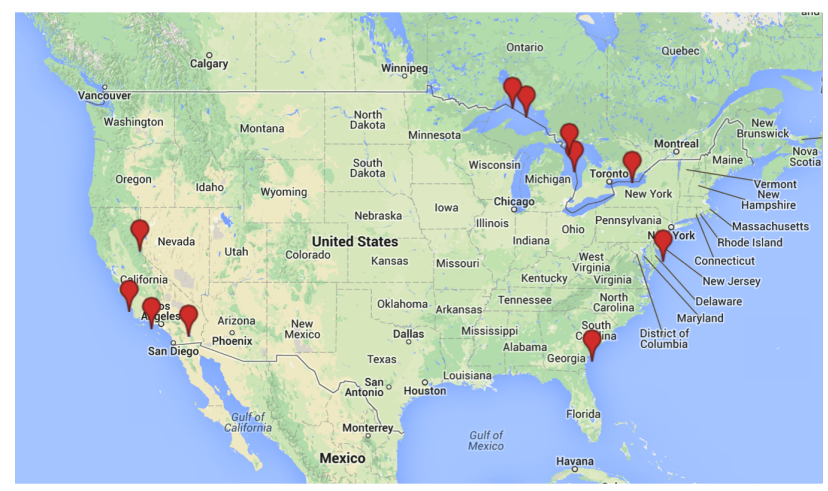

Figure 1: Distribution of validation sites throughout the United States.

negative value indicates an underestimation by our process. Figure 2 shows a histogram of error values for this cloud free Landsat 5 validation dataset.

$$
\text { Error }=\text { Predicted LST }- \text { Ground Truth }
$$

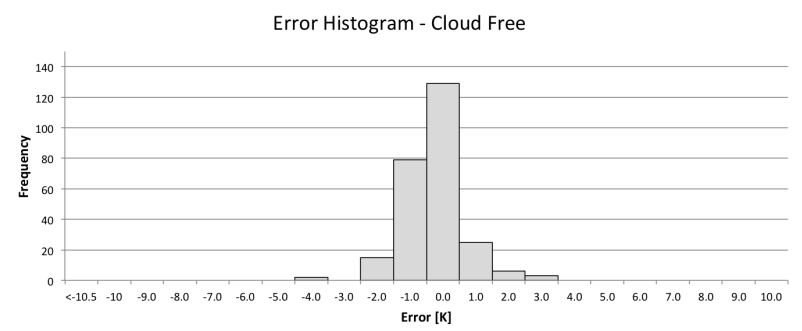

Figure 2: Error histogram for cloud free Landsat 5 validation dataset.

Note that for the applications at which this product is aimed, 1 $\mathrm{K}$ to $2 \mathrm{~K}$ errors are generally considered acceptable. Therefore, results from this histogram are extremely encouraging. The mean error for the 259 scenes shown is $-0.267 \mathrm{~K}$ and the standard deviation for this dataset is $0.900 \mathrm{~K} ; 90 \%$ of the dataset falls within the center three bins of the histogram with error values $[-1.5 \mathrm{~K}$, $1.5 \mathrm{~K}]$. These results are extremely encouraging and give us confidence in the datasources, tools, and chosen and implemented interpolations within the process. We do acknowledge that the dataset appears to have a slight negative bias, shown both by the negative mean error and also the slight left handedness of the histogram.

\section{CONFIDENCE METRIC DEVELOPMENT}

With confidence in the development and implementation of our proposed methodology, we realize that our goal is not only to process and produce results for cloud free scenes, but for all scenes, and all pixels, in the archive. All possible scenes with available ground truth data at the validation sites shown above over a given time span were downloaded and processed. This resulted in a dataset containing 827 images with comparable ground truth data. Initial investigations into a traditional error analysis, propagating expected error from input variables through the process, proved to be inaccurate for the proposed methodology. This traditional error analysis was impossible to implement in the presence or vicinity of clouds, pixels that we desire to process and characterize, and still difficult to accurately apply in cloud free pixels 
due to the inaccuracy of water vapor profiles (Cook and Schott, 2014). Because the presence of clouds is critical to understanding performance of the process, we turn to a more in depth cloud analysis to investigate performance of all scenes considered.

\subsection{Cloud Analysis}

Clouds are generally classified by height and texture. For height classifications, clouds are generally given the prefix cirro-, meaning high, or alto-, meaning mid. For texture classification, clouds are designated as strato-, meaning layered, uniform, widespread clouds, or cumulo-, meaning heaps of clouds or cellular, individual elements. From the view of the satellite, we are currently only concerned with the texture of the clouds and therefore create two categories: cumulus clouds, in which we group cirrocumulus, altocumulus, and other similar types, and stratus clouds, with which we group cirrostratus, altostratus, nimbostratus, and other similar types. Cirrus clouds are wispy, feathery clouds that are generally thinner but appear to exist more in layers, and are therefore included in the stratus cloud classification. For each image in the validation dataset, the image, and more specifically the area surrounding the ground truth data point, was visually analyzed and classified into one of the six categories shown in Table 1. It is important to realize that the categorization of type of cloud and in the vicinity are both subjective classifications based on the discretion of the analyst. However, all scenes in this dataset were categorized by the same analyst.

\begin{tabular}{|c|c|c|}
\hline Category & Description & Number of Scenes \\
\hline 0 & Cloud Free & $259[31.3 \%]$ \\
\hline 1 & Cumulus Vicinity & $98[11.9 \%]$ \\
\hline 2 & Stratus Vicinity & $158[19.1 \%]$ \\
\hline 3 & Cumulus at Pixel & $60[7.3 \%]$ \\
\hline 4 & Stratus at Pixel & $202[24.4 \%]$ \\
\hline 5 & Cloud Covered & $50[6.0 \%]$ \\
\hline
\end{tabular}

Table 1: Categories used in cloud analysis and breakdown of the number of scenes in each category for the Landsat 5 validation dataset.

\subsection{Cloud Analysis Results}

Figure 3 is a histogram of error values for all 827 images in the validation dataset. Figure 4 shows only scenes that were cloud free at the buoy or had clouds in the vicinity of the buoy, and Figure 5 shows only scenes that were cloud free at the buoy. Note that Figure 5 is the same as Figure 2 and repeated here for ease of comparison. The numbers in the plot title indicate the cloud categorizations of the scenes included in the plot.

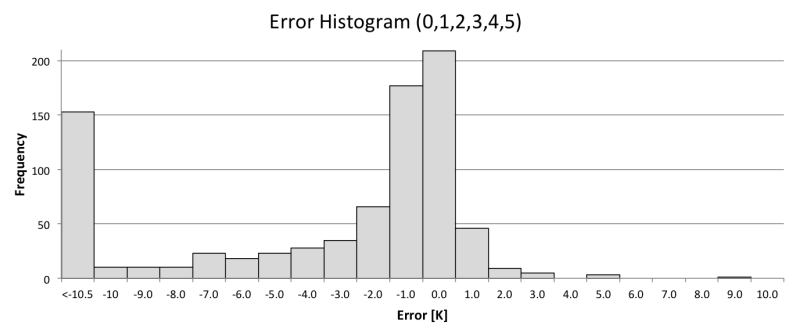

Figure 3: Error histogram for all images in Landsat 5 validation dataset.

Table 2 shows the mean and standard deviation of results as pixels with definite or likely cloud contamination are removed.

In Figure 3, the largest left hand bin is likely cloudy results, which we see is mostly eliminated in Figure 4 . The left hand tail per-

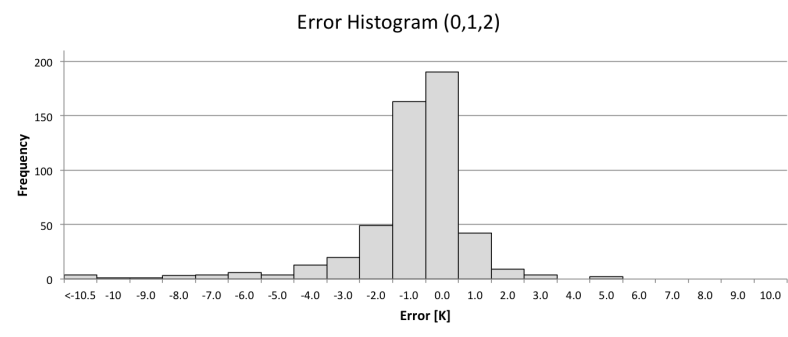

Figure 4: Error histogram for images in cloud categories 0, 1, and 2 in the Landsat 5 validation dataset.

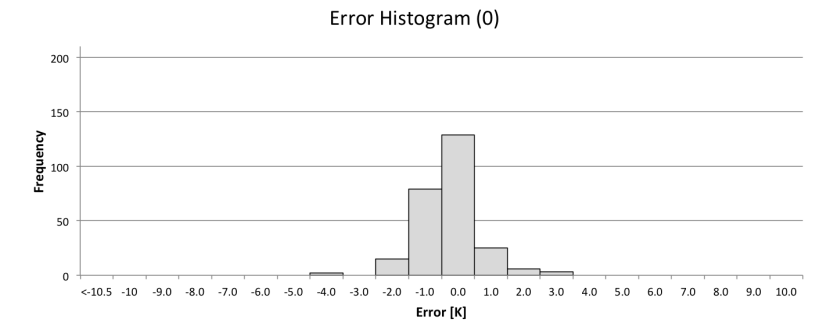

Figure 5: Error histogram including only scenes categorized as cloud free (0) from the Landsat 5 validation dataset.

sists in Figure 4, but note that nearly 30\% of the scenes are eliminated, and the number of scenes in the center three bins [1.5 $\mathrm{K}, 1.5 \mathrm{~K}$ ] decreases from 395 scenes to 233 scenes, when pixels with clouds in the vicinity are removed for Figure 5. This shows that although eliminating clouds in the vicinity eliminates the left hand tail shown in the histogram, it also eliminated a large portion (nearly $20 \%$ of the total dataset) of good scenes. We believe that some pixels with clouds in the vicinity could still be useful to many users, with an appropriate recognition of higher potential error.

\subsection{Cloud Product Incorporation}

For an operational product, obviously a visual analysis of each pixel is unreasonable. Incorporation of a cloud product, and automation of cloud categorization, is critical to the incorporation of the cloud analysis shown above. Landsat surface reflectance products are currently provided with a cloud mask; such a cloud mask should be provided with TM and ETM+, as well as Landsat 8 products, in the near future. Revisiting the subjective analysis shown above, we estimate a distance threshold of $0.5 \mathrm{~km}$ for cloudy pixels. That is, any ground truth site with clouds within $0.5 \mathrm{~km}$ (17 pixels) was classified as cloudy. Any ground truth site with clouds more than $0.5 \mathrm{~km}$ away but less than $5 \mathrm{~km}$ (167 pixels) away was classified as having clouds in the vicinity. Finally, any ground truth site with no clouds within $5 \mathrm{~km}$ was classified as cloud free. One example of a cloud product is shown in Figure 6 and classification of pixels as cloudy, clouds in the vicinity, or cloud free based on the distance thresholds described above and this cloud product is shown in Figure 7.

\subsection{Current Confidence Metric Suggestion}

Our current best suggestion for a confidence metric based on the atmospheric compensation to be included with the LST product is based on the cloud analysis and cloud influence shown above. For each image, an additional band will be included with the cloud categorizations, such as that shown in Figure 7. The expected mean and standard deviation for each category would also be included as shown in Table 3. Note that in Table 3, the means and 


\begin{tabular}{|c|c|c|c|}
\hline Cloud Category & Mean [K] & SD [K] & Number of Scenes \\
\hline $0,1,2,3,4,5$ & -8.471 & 19.313 & $826[100 \%]$ \\
\hline $0,1,2,3$ & -1.538 & 4.174 & $575[70 \%]$ \\
\hline $0,1,2$ & -0.933 & 2.460 & $515[62 \%]$ \\
\hline 0,1 & -0.499 & 2.228 & $357[43 \%]$ \\
\hline 0 & -0.267 & 0.900 & $259[31 \%]$ \\
\hline
\end{tabular}

Table 2: Mean and standard deviation for groups of results as scenes with definite and then likely cloud contamination are removed.

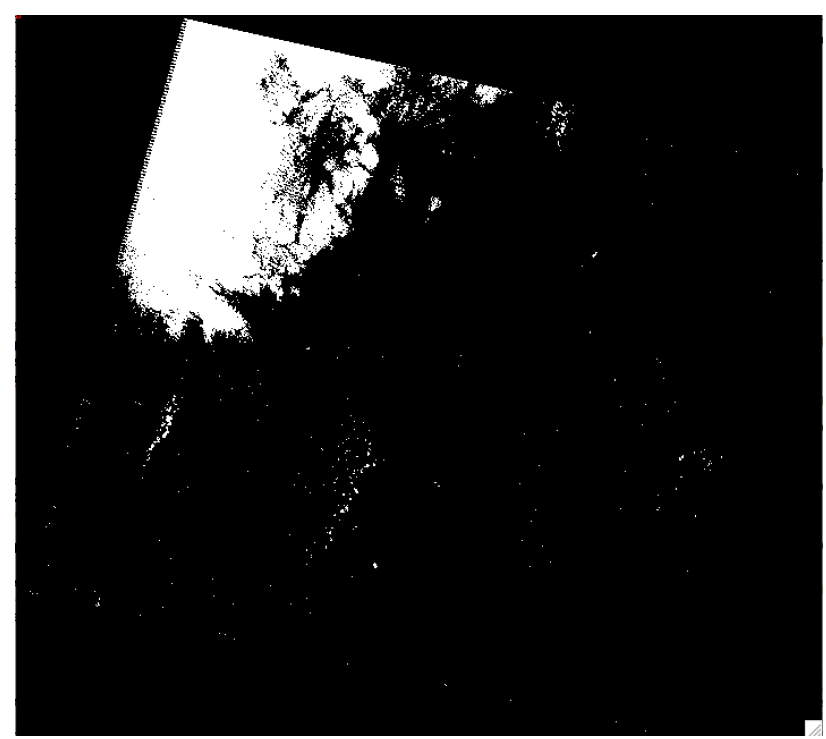

Figure 6: One example of a cloud product provided with the Landsat surface reflectance product. White pixels are classified as cloudy.

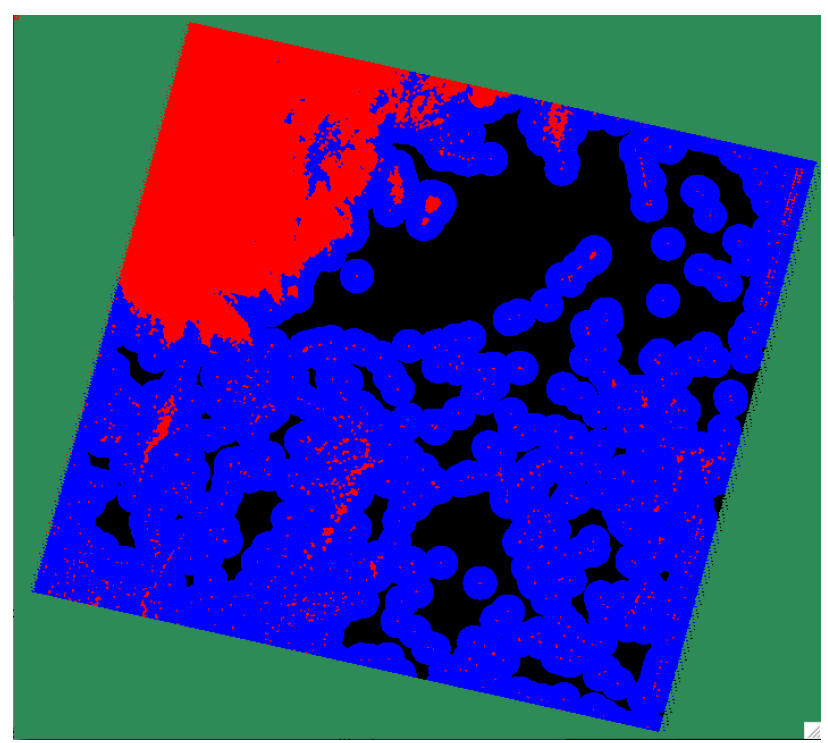

Figure 7: Example of a cloud product with each pixel categorized as cloudy (red), clouds in the vicinity (blue), or cloud free (black). Note that green pixels are pixels in the surround of the image.

standard deviations would be consistent for all images based on the validation dataset, but the scene percentage is unique to this example.

\begin{tabular}{|c|c|c|c|}
\hline Pixel & Mean [K] & SD [K] & \% of Scene \\
\hline Cloud Free (Black) & -0.267 & 0.900 & $13.2 \%$ \\
\hline Vicinity (Blue) & -1.607 & 3.239 & $65.8 \%$ \\
\hline Black and Blue & -0.933 & 2.460 & $86.8 \%$ \\
\hline Cloudy (Red) & Do Not Trust & NA & $21.0 \%$ \\
\hline
\end{tabular}

Table 3: Example of expected means and standard deviations for each category included with the additional confidence metric band.

\section{PRODUCT EXTENSIONS}

Two glaring holes in the ability of this product to be applied to every Landsat scene in the archive is the validation for other sensors as well as the extension to a global dataset. We briefly explore both in this section.

\subsection{Landsat 7}

A small subset of cloud free Landsat 7 scenes was processed over the same ground truth sites in order to validate the methodology for Landsat 7. The error was again calculated using Equation 9. A histogram of error values is shown in Figure 8. Only 44 cloud free Landsat 7 scenes were processed; these scenes had a mean error of $-0.20 \mathrm{~K}$ and a standard deviation of $0.68 \mathrm{~K}$. Although a larger dataset, with more variety in conditions and atmospheres, would be needed in order to more confidently validate Landsat 7 , this is a good first look, and results in similar mean errors when applying the methodology to more than one sensor. This is a very encouraging initial validation.

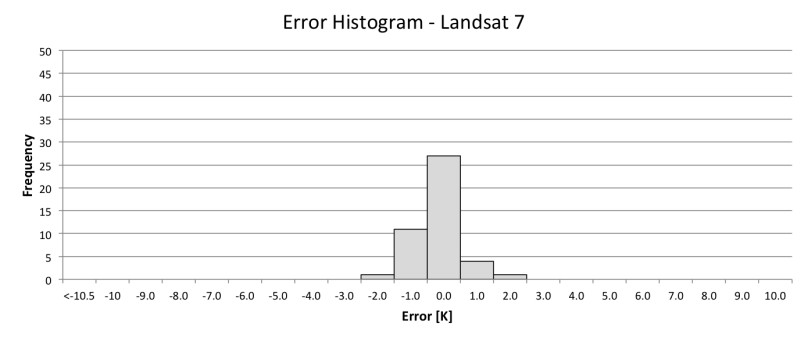

Figure 8: Histogram of error values for small subset of cloud free Landsat 7 scenes.

\subsection{Landsat 8}

Similarly, a small cloud free dataset of Landsat 8 scenes was processed and analyzed. Although Landsat 8 has two thermal bands, and could potentially utilize a split window or other multiple thermal band technique, we first analyze each band separately using our single channel method. Figure 9 shows a histogram of error values for band 10 of Landsat 8 and Figure 10 shows a histogram of error values for band 11 of Landsat 8 . The mean and standard deviation for each Landsat 8 band is summarized in Table 4 .

\begin{tabular}{|c|c|c|}
\hline Landsat 8 & Mean [K] & SD [K] \\
\hline Band 10 & -0.56 & 0.76 \\
\hline Band 11 & -2.16 & 1.64 \\
\hline
\end{tabular}

Table 4: Mean error and standard deviation for each band of Landsat 8 .

Band 10 has errors with magnitudes greater than those shown by Landsat 5 and Landsat 7, but the mean error and standard deviation are still less than $1 \mathrm{~K}$ and very reasonable for the targeted applications. However, the magnitude of errors for band 11 are more alarming and we would not be confident in producing a land surface temperature product using this radiance data. However, 


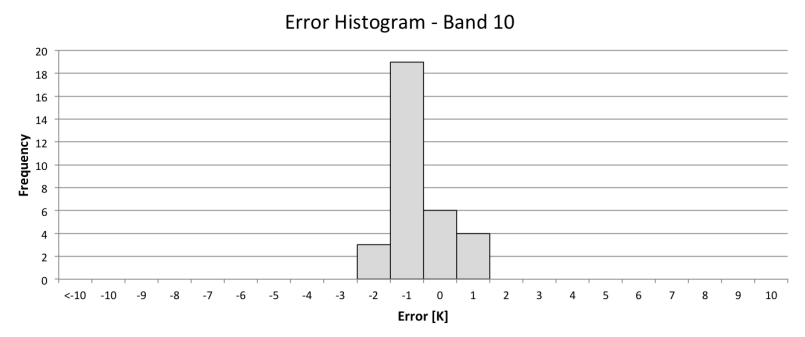

Figure 9: Histogram of error values for small subset of cloud free Landsat 8 scenes processed using band 10 .

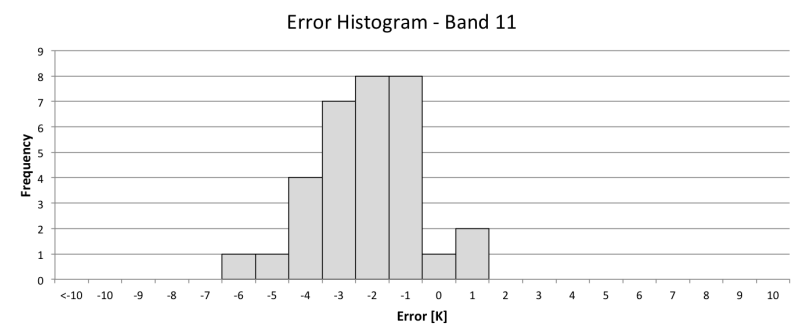

Figure 10: Histogram of error values for small subset of cloud free Landsat 8 scenes processed using band 11 .

current studies show that there are actual changes in the Landsat 8 calibration, more prevalent in band 11 ; it is believed that there is still currently a variable source of calibration error, correlated to scene radiance, that can be attributed to stray light from outside the detectors nominal point spread function impinging on the detector (Schott et al., 2014), (Montanaro et al., 2013). Therefore, these results, rather than acting as a reflection of the performance of our current methodology, will be utilized in the continuing calibration work and attempts to solve the stray light problem, and a more in depth analysis of the ability to process Landsat 8 using the current methodology will be revisited after there is more confidence in the calibration and characterization of the Landsat 8 thermal bands.

\subsection{Global Product}

As noted in Section 2.2, the current atmospheric characterization is pulled from a dataset that covers only North America. Extension to a global product requires utilization of global atmospheric characterization data. The Modern-Era Retrospective Reanalysis for research and Applications (MERRA) dataset provides $1.25^{\circ}$ resolution around the globe ( 288 by 144 array), has eight samples each day, and provides variables at 42 pressure levels. The MERRA data also includes geopotential height [gpm], which we convert to geometric height $[\mathrm{km}]$, and temperature $[\mathrm{K}]$, but provides the relative humidity [\%], eliminating the need for conversion of the water vapor profile (Rienecker and Gass, 2013). These profiles are integrated into the same methodology discussed in Section 3. A small subset of the Landsat 5 validation dataset, over the same ground truth sites, was processed using the MERRA reanalysis dataset. Histograms of error values for all scenes, scenes with clouds in the vicinity or cloud free, and only cloud free scenes are shown in Figures 11, 12, and 13 respectively. The numbers in the plot title indicate the cloud categorizations included in the plot. Note that the cloud analysis of these scenes was still subjective based on analyst interpretation and not objectively or automatically classified as described in the cloud product incorporation.

Table 5 shows the mean and standard deviation of the subset of

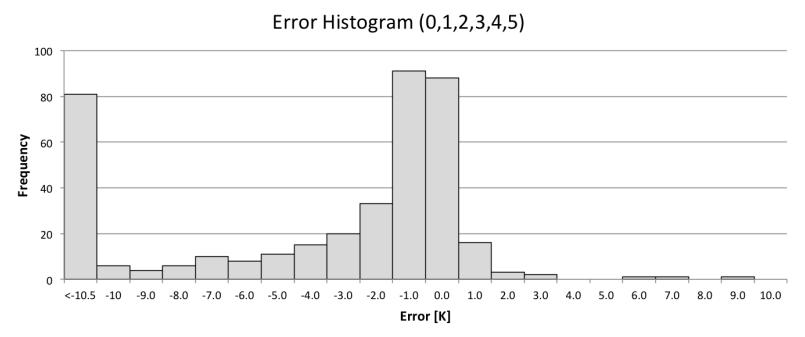

Figure 11: Histogram of error values for subset of Landsat 5 validation dataset processed using MERRA data.

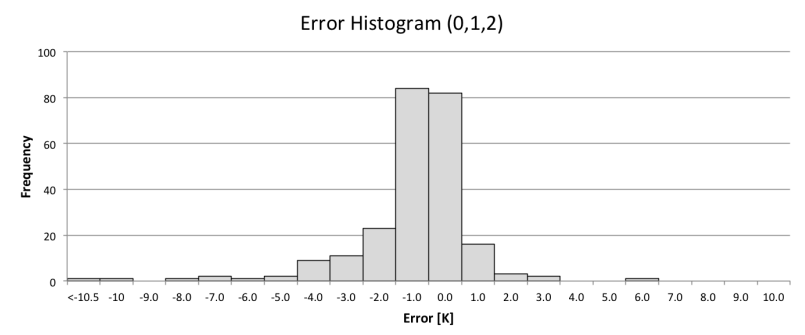

Figure 12: Histogram of error values for subset of Landsat 5 validation dataset processed using MERRA data, including only scenes with clouds in the vicinity and cloud free over the ground truth site.

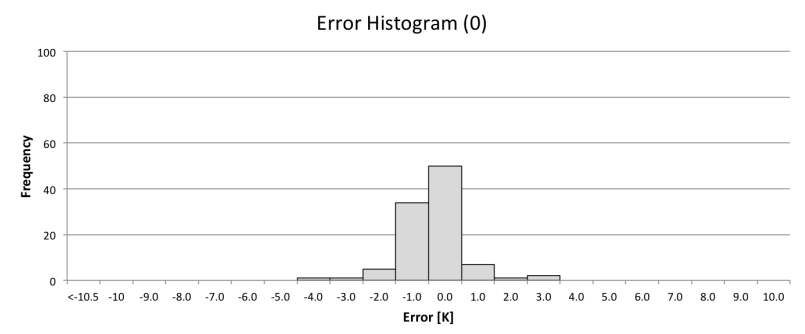

Figure 13: Histogram of error values for subset of Landsat 5 validation dataset processed using MERRA data, including only scenes that are cloud free over the ground truth site.

scenes, processed using MERRA. The values shown in Table 5 are similar in magnitude to those shown in Table 2.

Therefore, these results, although an incomplete analysis, are justification to move forward with a complete validation of the MERRA dataset for a global product and show very encouraging results for the ability to produce LST values for all Landsat scenes in the archive.

\section{FUTURE WORK}

\subsection{Bias Correction}

Comparison of current results for the cloud free Landsat 5 validation dataset shows the negative bias to be significant when compared to the Landsat 5 calibration data. Based on current calibration expectations for Landsat $5(0.0 \mathrm{~K} \pm 0.73 \mathrm{~K})$, results are significantly different at alpha levels 0.05 and 0.01 , suggesting that our negative bias is inherent to our process rather than the satellite data. Because we see a systematic bias, we suggest increasing each LST value by $0.267 \mathrm{~K}$ in order to create a zero mean in our cloud free data. The updated expected means and 


\begin{tabular}{|c|c|c|c|}
\hline Cloud Category & Mean $[\mathrm{K}]$ & SD [K] & Number of Scenes \\
\hline $0,1,2,3,4,5$ & -8.697 & 18.520 & $397[100 \%]$ \\
\hline $0,1,2,3$ & -1.474 & 3.610 & $262[66 \%]$ \\
\hline $0,1,2$ & -0.954 & 1.846 & $239[60 \%]$ \\
\hline 0,1 & -0.513 & 1.086 & $153[39 \%]$ \\
\hline 0 & -0.354 & 0.911 & $101[25 \%]$ \\
\hline
\end{tabular}

Table 5: Categories used in cloud analysis and breakdown of the number of scenes in each category for subset of scenes processed using MERRA dataset.

standard deviations for each category included in the confidence metric band are shown in Table 6 .

\begin{tabular}{|c|c|c|}
\hline Pixel & Mean [K] & SD [K] \\
\hline Cloud Free (Black) & 0.0 & 0.900 \\
\hline Vicinity (Blue) & -1.340 & 3.239 \\
\hline Black and Blue & -0.667 & 2.460 \\
\hline Cloudy (Red) & Do Not Trust & NA \\
\hline
\end{tabular}

Table 6: Expected means and standard deviations for each category included with the additional confidence metric band after bias correction for cloud free data.

However, analysis of our small Landsat 7 dataset shows the negative bias to be insignificant. That is, based on current Landsat 7 calibration expectations $(-0.05 \mathrm{~K} \pm 0.56 \mathrm{~K})$, results are not statistically different at alpha level 0.05 , suggesting any bias shown in these results cannot be solely attributed to our developed process. This would suggest that a bias correction to the final LST value would be inappropriate, although we recognize that this Landsat 7 dataset is also extremely small. Therefore, we intend to revisit the need for a bias correction after a more complete validation of all Landsat sensors.

\subsection{Cloud Product Automation}

The current confidence metric suggestion requires additional work before it can be implemented into the product. We must automate the incorporation of the cloud product and categorization of each pixel based on the distance thresholds given. With this automation, we can more accurately compare our objective and subjective cloud categorizations and adjust distance thresholds and expected means and standard deviations accordingly. Based on a larger dataset of automated results, we can explore further improvements. Would it be beneficial to bias pixels with clouds in the vicinity based on cloud type or distance to cloudy pixel? Is there a way to determine the type of cloud based on this cloud product and is that information useful for confidence expectations? We also need to address issues with single pixels classified as clouds and how to deal with the edges of each image.

\section{CONCLUSIONS}

We have shown a methodology to automatically generate the radiative transfer parameters necessary to calculate an LST value, given the corresponding surface emissivity, for every pixel in any Landsat scene in North America. This methodology shows extremely encouraging results for cloud free scenes, with a mean error when compared to ground truth data of $-0.267 \mathrm{~K}$, and a standard deviation of $0.900 \mathrm{~K}$. With this trusted methodology, we focus on the development of a confidence metric that will prove useful to the user. Based on limitations of an initial traditional error propagation when applied to this process, and the obvious and large influence of clouds in the scene, we found that a confidence metric based on the proximity of cloudy pixels would be best suited for this product. We currently suggest including an additional confidence metric band, derived from the Landsat cloud product, that categorizes each pixel as cloudy, clouds in the vicinity, or cloud free, with each category having some expected mean and standard deviation. Future work includes validation for all Landsat sensors and a global dataset, as well as incorporating the cloud product and improvements to the confidence metric band that could potentially provide the user with more information.

\section{ACKNOWLEDGEMENTS}

The authors would like to thank the Jet Propulsion Laboratory, NASA and USGS-EROS for their support of this work. This work is funded under NASA grant NMO710627, A Land Surface Temperature Product for Landsat.

\section{REFERENCES}

Barsi, J., Schott, J., Palluconi, F., Helder, D., Hook, S., Markham, B., Chander, G. and O'Donnell, E., 2003. Landsat tm and etm+ thermal band calibration. Can. J. Remote Sensing 29(2), pp. 141253.

Cook, M. J. and Schott, J. R., 2014. A novel confidence metric approach for a landsat land surface temperature product. In: ASPRS 2014 Annual Conference.

Hook, S. J., Vaughan, G., Tonooka, H. and Schladow, S. G., 2007. Absolute radiometric in-flight validation of mid infrared and thermal infrared data from aster and modis on the terra spacecradt using the lake tahoe, ca/nv, usa, automated validation site. IEEE Transactions on Geoscience and Remote Sensing 45(6), pp. 1798-1807.

Hulley, G. C. and Hook, S. J., 2009. The north american aster land surface emissivity database (naalsed) version 2.0. Remote Sensing of Environment 113(9), pp. 1967 - 1975.

Montanaro, M., Tesfaye, Z., Lunsford, A., Wenny, B., Reuter, D., Markham, B., Smith, R. and Thome, K., 2013. Preliminary onorbit performance of the thermal infrared sensor (tirs) on board landsat 8. Proc. SPIE 8866, Earth Observing Systems XVIII.

Padula, F. P., Schott, J. R., Barsi, J. A., Raqueno, N. G. and Hook, S. J., 2010. Calibration of landsat 5 thermal infrared channel: updated calibration history and assesment of the errors associated with the methodology. Can. J. Remote Sensing 36(5), pp. 617630.

Rienecker, M. and Gass, J., 2013. Merra: Modernera retrospective analysis for research and applications. http://gmao.gsfc.nasa.gov/research/merra/.

Schott, J. R., 2007. Remote Sensing: The Image Chain Approach. 2nd edn, Oxford Uniersity Press.

Schott, J. R., Raqueno, N., Montanaro, M., Ientilucci, E., Gerace, A. and Lunsford, A., 2014. Chasing the tirs ghosts: Calibrating the landsat 8 thermal bands. Proc. SPIE 9218, Earth Observing Systems XIX.

Shafran, P., 2007. North american regional reanalysis homepage. http://www.emc.ncep.noaa.gov/mmb/rreanl/.

Shepard, D., 1968. A two-dimensional interpolation function for irregularly-spaced data. ACM National Conference pp. 517-524.

SSI, 2012. About modtran. http://modtran5.com/about/index.html.

Wan, Z. and Dozier, J., 1996. A generalized split window algorithm for retrieving land-surface temperature from space. IEEE Transactions on Geoscience and Remote Sensing 34(4), pp. 892905. 